\title{
Phonon-Plasmon Interactions in Inhomogeneous Semiconductor Plasma Embedded with Nanoparticle Cluster
}

\author{
S. Ghosh* And P. Dubey \\ School of Studies in Physics, Vikram University, Ujjain, India \\ (Received August 19, 2017; in final form January 25, 2018)
}

\begin{abstract}
Phonon-plasmon interaction in inhomogeneous piezosemiconductor embedded with a nanoparticle cluster is examined using hydrodynamic model of plasma and macroscopic model of piezoelectric media. Present work dealt with the extensive investigation of acoustic wave amplification characteristics. The effects of density gradient $\delta$ and non-dimensional parameter $l$ related to nanoparticle cluster on acoustic gain have been studied with varying medium electron density $n_{0 e}$, wave frequency $\omega$ and velocity ratio $\vartheta_{0} / \vartheta_{s}$. The results so obtained, infer that the varying inhomogeneity and presence of nanoparticle cluster within the semiconductor plasma medium play decisive role in depicting the gain characteristics of acoustic wave.
\end{abstract}

DOI: $10.12693 /$ APhysPolA.133.1287

PACS/topics: 72.30.+q, 72.50.+b, 73.22.Lp

\section{Introduction}

Nanostructures and nanotechnology are one among the recent revolutionary developing fields of science and engineering. The ongoing research in this field is very relevant and is expected to substantially expand to competitively contribute to the solution of many grand challenges. They occupy the centre of scientific interest due to their extra ordinary optical and electrical properties $[1-3]$ as they are small enough to confine their electrons. This confinement of electrons in nanosized structures provide one of the most powerful and versatile means to control the electrical, optical, magnetic and thermoelectric properties of solid state functional materials. Even their presence within a material matrix is expected to be responsible for modifications in basic properties of bulk material.

In past couple of decades, the study of atomic and electronic properties of nanoparticles embedded within a GaAs matrix or within some other semiconductor matrix has caught the attention of material scientists. There were several theoretical as well as experimental reports on the growing mechanisms and some remarkable modifications in physical and chemical properties of such materials [4-9]. Using $\operatorname{In}_{0.53} \mathrm{Ga}_{0.47}$ As containing ErAs nanoparticles, Kim et al. [4] through their experimental and theoretical studies have demonstrated that the atomic substitution in alloys can efficiently scatter phonons, thereby reducing the thermal conductivity and correspondingly increasing thermoelectric figure of merit in crystalline solids. They concluded that while point defects in alloys efficiently scatter short wavelength phonons, the ErAs nanoparticles provide an additional scattering mechanism for the mid-to-long wavelength phonons.

*corresponding author; e-mail: drsanjayghosh.ssp@gmail.com
Zide et al. [5] have grown composite epitaxial materials that consist of semimetallic ErAs nanoparticles embedded in a semiconducting $\mathrm{In}_{0.53} \mathrm{Ga}_{0.47} \mathrm{As}$ matrix. They found that the presence of these particles increases the free electron concentration in the material while maintaining relatively high mobilities. The Seebeck coefficients measured for several sample structures are in reasonable agreement with experimentally observed values. They suggested that embedded metallic nanoparticles in a semiconductor matrix can serve as a building block for creating efficient thermoelectric materials. Kawasaki et al. [6] have examined the atomic and electronic structures of ErAs nanoparticles embedded within GaAs matrix via cross-sectional tunneling microscopy and spectroscopy. They found that the local density of states exhibits a finite minimum at the Fermi level demonstrating that the nanoparticles remain semimetallic despite the predictions of previous models of quantum confinement in ErAs.

Schultz and Palmstrom [7] have grown ErAs on GaAs surfaces that occurred by an embedded growth mode. They have shown that the surface morphologies obtained from their growth mode depend on structural differences, thermodynamics and diffusion. Lung and Marinescu [8] developed a phenomenological theory for cross plane transport in a semiconductor superlattice doped with nanostructures to study the improvement in the thermoelectric properties. They have reported about $20 \%$ increase in the value of the thermopower measured.

A theoretical analysis on thermoelectric figure of merit enhanced by nanostructuring of thermoelectric materials was presented by Choudhary [9]. He has reported significant modifications in all thermal parameters of the system due to embedded nanoparticles. Even though majority of these works are confined to thermoelectric properties of these materials, they suggest that nanoparticle embedded semiconductor matrix is a very interesting medium to be studied in detail. This type of media is now becoming friendlier to technologists because of 
its utility in processing of new materials; those are useful in fabrication of improved devices. But, we found that the study of wave particle interactions in these media is still untouched. Based on this, in some of our previous reports, we have investigated electron-electron two-stream interactions in homogeneous semiconductor ( $n$-GaAs) plasma embedded with single nanoparticle [10] and assembly of nanoparticle cluster [11]. We found that the presence of nanoparticles interestingly modifies the two-stream space charge gain characteristics within the homogeneous semiconductor plasma medium.

If an acoustic wave propagates through semiconductor plasma medium, it may interact with various elementary excitations. One of these excitations is plasmon and its interaction with conduction electrons is of considerable interest. In such an interaction the acoustic wave may lose or gain energy under certain physical conditions. The former process is known as attenuation and latter as amplification of acoustic wave. The possibility of acoustic amplification has been first realised in piezoelectric semiconductor by applying a dc electric field [12]. When electron drift induced by external electric field exceeds the sound velocity (i.e., $\vartheta_{0}>\vartheta_{s}$ ), the acoustic wave amplification occurs due to phonon emission by carriers [1315]. Motivated by this, a number of researchers worked in this direction and explored various methods of acoustic amplification in different semiconductors [16-18].

We know that, under crossed field configurations, complete homogeneity of a semiconductor system cannot be achieved. Otherwise, also the inhomogeneity may be induced in any semiconductor material by non-uniform doping or by exposing it to non-uniform radiation. It is very difficult to grow a complete homogeneous semiconductor experimentally. Now, if the system has gradients of different physical parameters of the medium, a plasma current or a particle drift exists; otherwise in the presence of electric field the gradients enhance the particle drift. As we have discussed above, this particle drift is the only parameter which actually decides the amplification/attenuation of the acoustic wave while propagating through a semiconductor medium. Recently, many investigators have reported their efforts of investigating acoustic wave amplification in homogeneous [19-21] as well as inhomogeneous [22-25] semiconductor plasma medium under several physical regimes and field geometries.

We found that the problem of how carrier density gradient and presence of nanoparticle cluster influence phonon-plasmon coupling and resultantly, the gain characteristic of the acoustic wave in presence of nanoparticle cluster within an inhomogeneous semiconductor has not been reported yet. Hence, in the present report we have discussed our study on the possible signature of density gradient and assembly of nanoparticle cluster on phononplasmon interactions in a wide band gap piezoelectric semiconductor ( $n$-CdS) plasma medium embedded with nanoparticle cluster. We hope that this report may be the very first attempt in this direction.

\section{Theoretical formulation}

When a piezoelectric semiconductor doped with a cluster of nanoparticles is imposed upon by an external dc electric field, the free electrons from conduction band of the semiconductor acquire drift velocity in opposite direction to that of applied electric field, whereas the electrons present in the cloud of the nanoparticle cluster are displaced from their equilibrium position. As per fluid dynamics, the displacement velocity of electron cloud adds vectorially with drift velocity of free electrons of semiconductor and results into an average velocity of electron. On the other side, because of the resulted restoring force due to displacement in electron cloud of nanoparticle cluster, these electrons start collective oscillations identical to plasma oscillations of free electrons of semiconductor medium. This phenomenon resulted into a stiffer spring constant that leads to a modified plasma frequency. From the coupled mode view point, since the synchronism between the phonon mode and plasmon occurs at electron plasma frequency, one may safely infer that the center of interaction frequency band will be modified because of modification in electron plasma frequency. These two phenomena invoked due to the presence of nanoparticle cluster may be attributed for the expected modifications in the amplification characteristic of acoustic wave.

In order to study the effect of density gradient and nanoparticle cluster on phonon-plasmon interaction in $n$-type piezoelectric semiconductor plasma medium ( $n$ CdS) embedded with an assembly of nanoparticle cluster, we consider that this nanoparticle cluster has particle number density $N$, electron cloud density $n_{0 n}$, and average radius of the particle $r$. The medium is subjected to an applied electrostatic field $\boldsymbol{E}_{0}$ along negative $z$-direction, hence the medium electrons having electron density $n_{0 e}$, get drifted in positive $z$-direction with velocity $\boldsymbol{\vartheta}_{0}$. We assume that this inhomogeneous semiconductor plasma medium has the density gradient $\nabla n_{0 e}$ along $z$-direction.

Here, we consider that the medium belongs to cubic symmetry group that simplifies the involved tensor components without diluting the interaction under study. We assumed that the sound wave is a shear wave propagating along $z$-axis which is (011) axis of the crystal. The lattice displacement $\boldsymbol{u}$ of shear acoustic wave is assumed to be only in $x$-direction. This geometry is appropriate to many compound semiconductors belonging to class 31 and exhibit piezoelectricity [26]. The dielectric tensor of this crystal class is always diagonal and isotropic.

We will use the concept of displacement vector and vector of a given point on the lattice, to describe the acoustic wave in crystals. Now, the equation of lattice vibrations for the considered field geometry may be written as

$$
\rho \frac{\partial^{2} u_{x}(z, t)}{\partial t^{2}}=\frac{\partial T_{13}}{\partial z}
$$

in which $\rho$ is the mass density of the medium and $T_{13}$ is the component of elastic stress tensor. Since, here acous- 
tic wave propagates along a particular axis of symmetry in a cubic crystal, one may very safely avoid the tensor notations. Then Eq. (1) transforms into a scalar one and may be written as

$$
\rho \frac{\partial^{2} u_{x}(z, t)}{\partial t^{2}}=C_{44} \frac{\partial^{2} u_{x}(z, t)}{\partial z^{2}} .
$$

Here, $C_{44}$ is the two-index designation of the elastic modulus in cubic crystal which as per Hooke's law determines the relation between stress and linear deformation as

$$
T_{13}=T_{31}=2 C_{44} S_{13}=2 C_{44} \frac{\partial u_{x}(z, t)}{\partial z} .
$$

There are two other independent components $C_{11}$ and $C_{12}$ which do not participate in this shear wave problem.

To derive Eq. (3), the relation between strain and lattice displacement is used which may be written as

$$
S_{13}=S_{31}=\frac{1}{2} \frac{\partial u_{x}(z, t)}{\partial z} .
$$

Due to piezoelectric nature of the crystals, appearance of electric field is due to mechanical stress and vice versa. For these piezoelectric crystals, Eq. (2) may be supplemented by the relation between electric field and elastic stress given by

$$
T_{13}=T_{31}=-\beta E_{z}(z, t) .
$$

Here, $\beta$ is the piezoelectric coefficient of the medium and $E_{z}(z, t)$ is the piezoelectric field. To simplify the problem without neglecting any important observation, here we have assumed that in our case elastic moduli and piezoelectric coefficients are scalar everywhere.

Now, Eq. (1) can be re-written as

$$
\rho \frac{\partial^{2} u_{x}(z, t)}{\partial t^{2}}=C_{44} \frac{\partial^{2} u_{x}(z, t)}{\partial z^{2}}-\beta \frac{\partial E_{z}(z, t)}{\partial z} .
$$

Assuming a perturbation $E_{1 z}=\tilde{E}_{1 z} \exp (\mathrm{i}(\omega t-k z))$ is imposed on the medium, in which $(\omega, k)$ are wave frequency and wave number involved. Following Steele and Vural [27], the wave equation in an elastic piezoelectric medium may be written as

$$
\left(-\rho \omega^{2}+C_{44} k^{2}\right) \tilde{u}_{x}=\mathrm{i} k \beta \tilde{E}_{z} .
$$

In a non-piezoelectric $(\beta=0)$ material the above Eq. (7) represents the usual shear acoustic wave propagation in a cubic crystal, with acoustic speed $\vartheta_{s}\left(=\sqrt{\frac{C_{44}}{\rho}}\right)$. While for piezoelectric $(\beta \neq 0)$ crystal, Eq. ( 7$)$ infers that the acoustic wave gets coupled with the electron motion through the piezoelectric field $\tilde{E}_{z}$. The semiconductor plasma always exhibits a tendency to screen piezoelectric field and thereby to change the piezoelectric deformation. This tendency actually connects the perturbations in the semiconductor plasma medium with those of the lattice.

Using Eq. (7) and usual stress and electric displacement components of cubic crystals, one obtains

$$
\tilde{D}_{z}=\varepsilon \tilde{E}_{z}\left(1+\frac{\beta^{2} k^{2}}{\varepsilon\left(-\rho \omega^{2}+C_{44} k^{2}\right)}\right),
$$

where $\varepsilon\left(=\varepsilon_{0} \varepsilon_{L}\right)$ is the permittivity of the medium, in which $\varepsilon_{0}$ and $\varepsilon_{L}$ are permittivity of free space and lattice dielectric constant of the semiconductor material considered here.
Equation (7) describes the first of the interacting waves and the corresponding total induction is expressed in Eq. (8). The second wave is the one sustained by the charge flux in the semiconductor nanoparticle cluster matrix considered here as medium of study.

To deal with the electrokinetic mode, which is the second wave of the interaction under study, let us assume that the motion of the free electrons of the conduction band of cubic piezoelectric semiconductor plasma medium is controlled by the hydrodynamic model of plasmas. Now, if collisions with the lattice and diffusion (thermal velocity) are taken into account, the onedimensional momentum transfer and continuity equations may be expressed as

$$
\begin{aligned}
& \left(\mathrm{i}\left(\omega-k \vartheta_{0}\right)+\nu_{e}\right) \tilde{\vartheta}_{1 z}= \\
& \quad(-e / m) \tilde{E}_{1 z}+i \vartheta_{\theta}^{2}\left(n_{1} / n_{0 e}\right) \tilde{k}, \\
& \left(\frac{n_{1}}{n_{0 e}}\right)=\frac{\vartheta_{1 z}(k+i \delta)}{\omega-\vartheta_{0}(k-i \delta)} .
\end{aligned}
$$

Here, $\delta=\nabla n_{0 e} / n_{0 e}$.

By solving this basic one-dimensional momentum transfer Eq. (9) for a small harmonic perturbation in the linear approximation, the perturbed velocity $\left(\vartheta_{1 z}\right)$ of free electrons of the semiconductor medium under quasistatic limit $\left(k^{2} C_{L}^{2} \gg \omega^{2}, C_{L}\right.$ being the speed of light in the material) may be obtained as

$$
\tilde{\vartheta}_{1 z}=\frac{\mathrm{i}(e / m)}{F_{1}(\omega, k)} \tilde{E}_{z},
$$

where

$$
F_{1}(\omega, k)=\omega-k \vartheta_{0}-\mathrm{i} \nu_{e}-\frac{D_{\theta} \nu_{e} k^{2}(1+\mathrm{i} \delta / k)}{\omega-k \vartheta_{0}\left(1-\mathrm{i}^{\delta} / k\right)},
$$

in which $\nu_{e}$ is the momentum transfer collision frequency and $D_{\theta}\left(=\frac{\vartheta_{\theta}^{2}}{\nu_{e}}\right)$ is the thermal diffusion coefficient, $\vartheta_{\theta}$ being the electron thermal velocity of the semiconductor medium.

Now, conduction current density for free electron of the medium is given by

$$
\tilde{J}=-e n \vartheta .
$$

Thus, first-order conduction current density may be obtained as

$$
\tilde{J}_{1}=-e\left(n_{0 e} \vartheta_{1}+n_{1} \vartheta_{0}\right) .
$$

As electric field is applied along $z$-direction, the conduction current density reduces to

$$
\tilde{J}_{1 z}=-e n_{0 e}\left(\vartheta_{1 z}+\frac{n_{1}}{n_{0 e}} \vartheta_{0}\right) .
$$

Using Eqs. (10) and (11) in Eq. (12), we may obtain the conduction current density for free electrons of the semiconductor medium as

$$
\tilde{J}_{1 z}=-\mathrm{i} \varepsilon \omega_{p e}^{2} \frac{\omega+2 \mathrm{i} \delta \vartheta_{0}}{F_{1}(\omega, k)\left(\omega-k \vartheta_{0}+\mathrm{i} \delta \vartheta_{0}\right)} \tilde{E}_{z} .
$$


Now, the motion of electrons present within the nanoparticle cloud having displacement $\Delta$ under the influence of applied electrostatic field $\boldsymbol{E}_{0}$ can be described by the following equation of motion [28]:

$$
\frac{\mathrm{d}^{2} \Delta}{\mathrm{d} t^{2}}+\frac{\omega_{p n}^{2}}{3} \Delta=-\frac{e E_{z}(z, t)}{m} .
$$

Here, $\omega_{p n}=\sqrt{e^{2} n_{0 n} / m \varepsilon}$ is the plasma frequency of electrons present within the cloud of nanoparticle cluster. Again solving Eq. (14) for small harmonic perturbations under linear approximation, we may get the velocity of electron cloud of nanoparticle cluster as

$$
\tilde{\vartheta}_{n p}=\frac{i \omega(e / m) \tilde{E}_{z}}{\left(\omega^{2}-\frac{\omega_{p n}^{2}}{3}\right)} .
$$

We know that the first order current density for electron cloud of nanoparticle cluster may be expressed as

$$
\tilde{J}_{n p}=-\frac{4 \pi}{3} r^{3} N n_{0 n} e \tilde{\vartheta}_{n p} .
$$

Combining Eqs. (15) and (16), we get that the expression for the current density related to electron cloud of nanoparticle cluster becomes

$$
\tilde{J}_{n p}=-\mathrm{i} \varepsilon \omega \frac{4 \pi}{3} l \frac{\omega_{p n}^{2}}{\omega^{2}-\omega_{p n}^{2} / 3} \tilde{E}_{z} .
$$

Here, $l=r^{3} N$ is a non-dimensional physical parameter related to nanoparticle cluster.

Using Eqs. (13) and (17), the resultant current density $\tilde{J}_{1}=\tilde{J}_{1 z}+\tilde{J}_{n p}$ in considered system becomes

$$
\begin{aligned}
\tilde{J}_{1} & =-\mathrm{i} \varepsilon\left(\frac{\omega_{p e}^{2}}{F(\omega, k)} \frac{\omega+2 \mathrm{i} \delta \vartheta_{0}}{\omega-k \vartheta_{0}+\mathrm{i} \delta \vartheta_{0}}\right. \\
& \left.+\frac{4 \pi}{3} l \omega \frac{\omega_{p n}^{2}}{\left(\omega^{2}-\omega_{p n}^{2} / 3\right)}\right) \tilde{E}_{z} .
\end{aligned}
$$

Combining this resultant current density $\tilde{J}_{1}$ (Eq. (18)) with continuity Eq. (10), the resultant space charge density $\tilde{n}_{1}$ may be deduced as

$$
\begin{aligned}
\tilde{n}_{1} & =\frac{\mathrm{i} \varepsilon k}{e\left(\omega-k \vartheta_{0}\right)}\left(\frac{\omega_{p e}^{2}}{F(\omega, k)} \frac{\omega+2 i \delta \vartheta_{0}}{\omega-k \vartheta_{0}+\mathrm{i} \delta \vartheta_{0}}\right. \\
& \left.+\frac{4 \pi}{3} l \omega \frac{\omega_{p n}^{2}}{\omega^{2}-\omega_{p n}^{2} / 3}\right) \tilde{E}_{z} .
\end{aligned}
$$

Substitution of Eq. (19) in Maxwell's equation $\nabla \cdot \vec{D}=-e n_{1}$, derives the electric displacement for free electrons of the semiconductor nanoparticle cluster matrix and that may be expressed as

$$
\begin{aligned}
\tilde{D}_{z} & =\frac{\varepsilon \tilde{E}_{z}}{\omega-k \vartheta_{0}}\left(\frac{\omega_{p e}^{2}}{F(\omega, k)} \frac{\omega+2 \mathrm{i} \delta \vartheta_{0}}{\omega-k \vartheta_{0}+\mathrm{i} \delta \vartheta_{0}}\right. \\
& \left.+\frac{4 \pi}{3} l \omega \frac{\omega_{p n}^{2}}{\omega^{2}-\omega_{p n}^{2} / 3}\right) .
\end{aligned}
$$

Comparison of Eqs. (8) and (20) yields the general dispersion relation for phonon-plasmon interaction in inhomogeneous semiconductor medium embedded with a nanoparticle cluster as

$$
\begin{aligned}
& \left(\omega^{2}-k^{2} \vartheta_{s}^{2}\right)\left[1-\frac{1}{\omega-k \vartheta_{0}}\right. \\
& \times\left(\frac{\omega_{p e}^{2}\left(\omega-k \vartheta_{0}\left(1-\mathrm{i}^{\delta} / k\right)\right)\left(\omega+2 \mathrm{i} \delta \vartheta_{0}\right)}{\left(\omega-k \vartheta_{0}-\mathrm{i} \nu\right)\left(\omega-k \vartheta_{0}\left(1-\mathrm{i}^{\delta} / k\right)\right)-D_{\theta} \nu_{e} k^{2}(1+\mathrm{i} \delta / k)}\right. \\
& \left.\left.\times \frac{1}{\omega-k \vartheta_{0}+\mathrm{i} \delta \vartheta_{0}}+\frac{4 \pi}{3} l \omega \frac{\omega_{p n}^{2}}{\left(\omega^{2}-\omega_{p n}^{2} / 3\right)}\right)\right]=K^{2} k^{2} \vartheta_{s}^{2} .
\end{aligned}
$$

The term in first bracket on LHS corresponds to the usual shear acoustic mode and term in second bracket corresponds to the plasmon mode modified due to the presence of a nanoparticle cluster via electron cloud plasma frequency $\left(\omega_{p n}\right)$ and density gradient via $\delta$ term. RHS contains the electromechanical coupling coefficient $K^{2}\left(=\beta^{2} / C_{44} \varepsilon\right)$ generated due to piezoelectric nature of the crystal. If the medium is non-piezoelectric $(\beta=0)$, this coupling coefficient vanishes from Eq. (21) and results in two independent modes of propagation. These modes may be expressed as

$\left(\omega^{2}-k^{2} \vartheta_{s}^{2}\right)=0$,

$1-\frac{1}{\omega-k \vartheta_{0}}$

$\times\left(\frac{\omega_{p e}^{2}\left(\omega-k \vartheta_{0}\left(1-\mathrm{i}^{\delta} / k\right)\right)\left(\omega+2 \mathrm{i} \delta \vartheta_{0}\right)}{\left(\omega-k \vartheta_{0}-\mathrm{i} \nu\right)\left(\omega-k \vartheta_{0}\left(1-\mathrm{i}^{\delta} / k\right)\right)-D_{\theta} \nu_{e} k^{2}\left(1+\mathrm{i}^{\delta} / k\right)}\right.$

$\left.\times \frac{1}{\omega-k \vartheta_{0}+\mathrm{i} \delta \vartheta_{0}}+\frac{4 \pi}{3} l \omega \frac{\omega_{p n}^{2}}{\left(\omega^{2}-\omega_{p n}^{2} / 3\right)}\right)=0$.

Equation (22a) represents usual shear mechanical mode propagation through an elastic medium and Eq. (22b) stands for the plasma/electrokinetic mode whose usual nature has been modified due to the presence of nanoparticle cluster and density gradient within the host medium.

Following Steele and Vural [27], we have solved the derived dispersion relation (Eq. (21)), under collision dominated limit $\left(\nu_{e} \gg \omega, k \vartheta_{0}\right)$, using the standard approximation $\left(k \vartheta_{s} / \omega\right)=1+i \alpha$; where $\alpha(\ll 1)$ is gain per radian. By assuming that the spatial rate of change of static quantity is much smaller than time varying quantity $|\vec{\delta}| \ll|\vec{k}|$, we obtain the expression for gain per radian as

$$
\begin{aligned}
\alpha= & \frac{1}{2} K^{2} \omega^{3} \omega_{R} \gamma\left(\gamma-\frac{\delta \vartheta_{0}}{\omega_{D}}\right)\left[\left(\omega \gamma\left(\omega \gamma+\frac{4 \pi}{3} l \omega A\right)\right.\right. \\
& \left.\left.+2 \omega_{R} \delta \vartheta_{0}\right)^{2}-\left(D_{\theta} k^{2}\left(\omega \gamma+\frac{4 \pi}{3} l \omega A\right)-\omega \omega_{R}\right)^{2}\right]^{-1} .
\end{aligned}
$$

Here, $\gamma=\vartheta_{0} / \vartheta_{s}-1, \omega_{D}=\vartheta_{s}^{2} / D_{\theta}, \omega_{R}=\omega_{p e}^{2} / \nu_{e}$ and $A=\omega_{p n}^{2} /\left(\omega^{2}-\omega_{p n}^{2} / 3\right)$. The term $A$ in Eq. (23) represents the modification in acoustic gain due to the nanoparticle cluster via the plasma frequency $\left(\omega_{p n}\right)$ of electron cloud of nanoparticle cluster. The term $\delta$ is responsible for modification in acoustic gain due to inhomogeneity in the medium. The sound wave is said to be amplified when $\alpha>0$ and attenuating when $\alpha<0$. 


\section{Results and discussion}

Our principal aim is to present numerical demonstration of gain profiles of acoustic wave in a piezoelectric semiconductor embedded with nanoparticle cluster and to investigate possible signature of density gradient on it. For this purpose, we have considered $n$-CdS embedded with nanoparticle cluster at room temperature represented by the following physical parameters:

$m_{e}=0.17 m_{0}, \quad \varepsilon_{L}=9.35, \quad \beta=0.21 \mathrm{Cm}^{-2}$,

$\mu=0.035 \mathrm{~m}^{2} \mathrm{~V}^{-1} \mathrm{~s}^{-1}$ and $\rho=4820 \mathrm{~kg} \mathrm{~m}^{-3}$.

On solving Eq. (23) using these physical parameters, the dependence of acoustic gain $\alpha$ on carrier density $n_{0 e}$, wave frequency $\omega$, and velocity ratio $\vartheta_{0} / \vartheta_{s}$ have been estimated numerically and are depicted in Figs. 1-5.

Figure 1 represents the variation of acoustic gain $\alpha$ with free electron density $n_{0 e}$ in presence of nanoparticle cluster using density gradient $\delta$ as parameter. Curves in all the three cases infer that at low doping regime acoustic mode is amplifying in nature. In this regime the amplification coefficient is a parabolically increasing function of free electron density $n_{0 e}$. At a particular value of $n_{0 e}$ gain coefficient attains a maximum and then suddenly reduces to zero. On increasing $n_{0 e}$ further, acoustic mode starts attenuating sharply with increasing attenuating coefficient. The attenuation coefficient acquires maxima and afterwards starts reducing exponentially with electron density $n_{0 e}$. This propagating mode becomes stable in highly doped medium. Thus, we may safely conclude that moderately doped semiconductor medium is the best

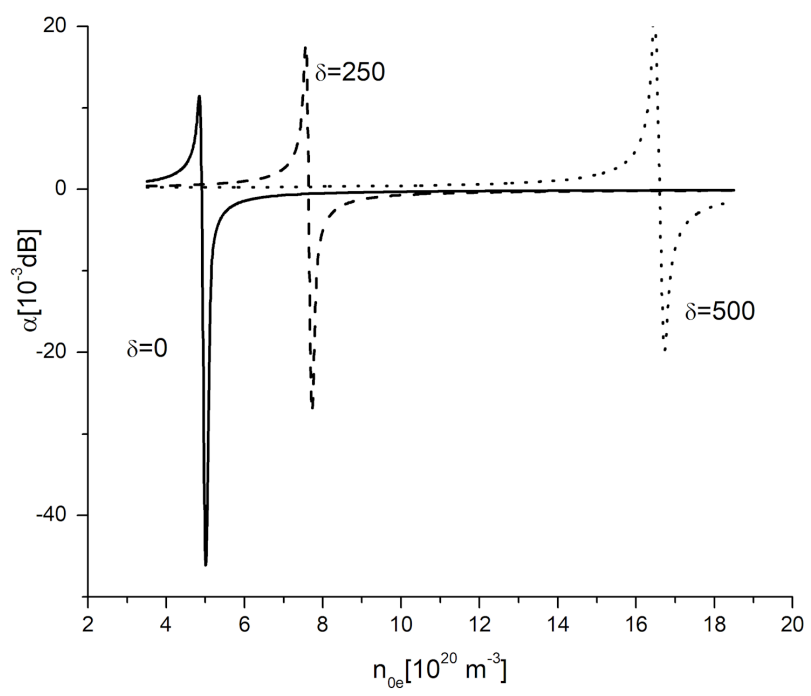

Fig. 1. $\alpha$ vs $n_{0 e}$ for different $\delta$ at $l=0.001$.

host to achieve sound amplification. This figure also infers that the amplification to attenuation crossover point is shifted to higher values of electron density on increasing the inhomogeneity parameter $\delta$.
Figure 2 depicts the gain per radian $\alpha$ versus free electron density $n_{0 e}$ character for $\delta=100$ at different values of non-dimensional parameter $l$. It is again clear from this figure that the low doped semiconductor medium is favourable for amplifying acoustic mode. The amplification coefficient enhances slowly with $n_{0 e}$ and reaches its peak value at $n_{0 e} \approx 5.6 \times 10^{20} \mathrm{~m}^{-3}$. On further increasing $n_{0 e}$, the amplification coefficient reduces sharply, crosses zero value and then changes its character to attenuating nature. This attenuation coefficient rapidly increases to its maximum value at $n_{0 e} \approx 5.8 \times 10^{20} \mathrm{~m}^{-3}$. With further increase in $n_{0 e}$, attenuation coefficient sharply reduces to zero to make the mode stable at $n_{0 e} \approx 6 \times 10^{20} \mathrm{~m}^{-3}$. Form both figures (1 and 2$)$, it may be inferred that on increasing the number of nanoparticles in the cluster and/or the inhomogeneity in the medium, the magnitudes of maximum amplification/attenuation coefficients increase/decrease.

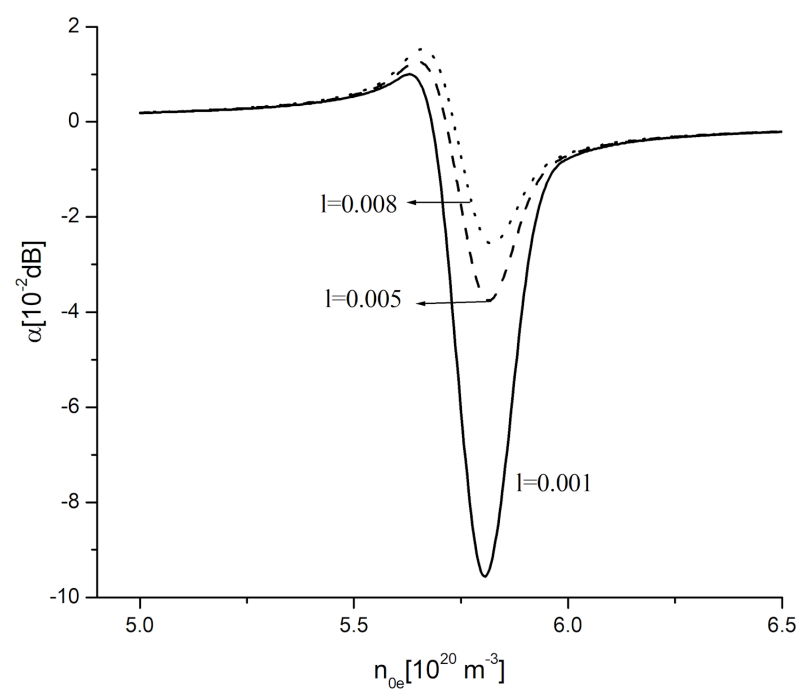

Fig. 2. $\alpha$ vs $n_{0 e}$ for different $l$ at $\delta=100$.

The nature of variation of acoustic gain $\alpha$ against wave frequency $\omega$ with $\delta$ as parameter for a nanoparticle cluster embedded semiconductor medium is shown in Fig. 3. In a homogeneous $\delta=0$ medium, the acoustic mode is attenuating with rapidly increasing attenuation coefficient that acquires maximum at $\omega \approx 3 \times 10^{7} \mathrm{~s}^{-1}$. A slight tuning at this wave frequency yields that the nature of the acoustic mode is converted from decaying to amplifying, whose amplification coefficient immediately touches its maximum. After this value of wave frequency the acoustic mode becomes stable. On the other hand, for inhomogeneous medium $\delta \neq 0$ the acoustic mode is found to be amplifying throughout the frequency range under study. From curves of this figure, it may also be inferred that the increase in inhomogeneity shifts the peak of the gain characteristics to higher wave frequency side, but reduces the overall magnitude of acoustic gain. 


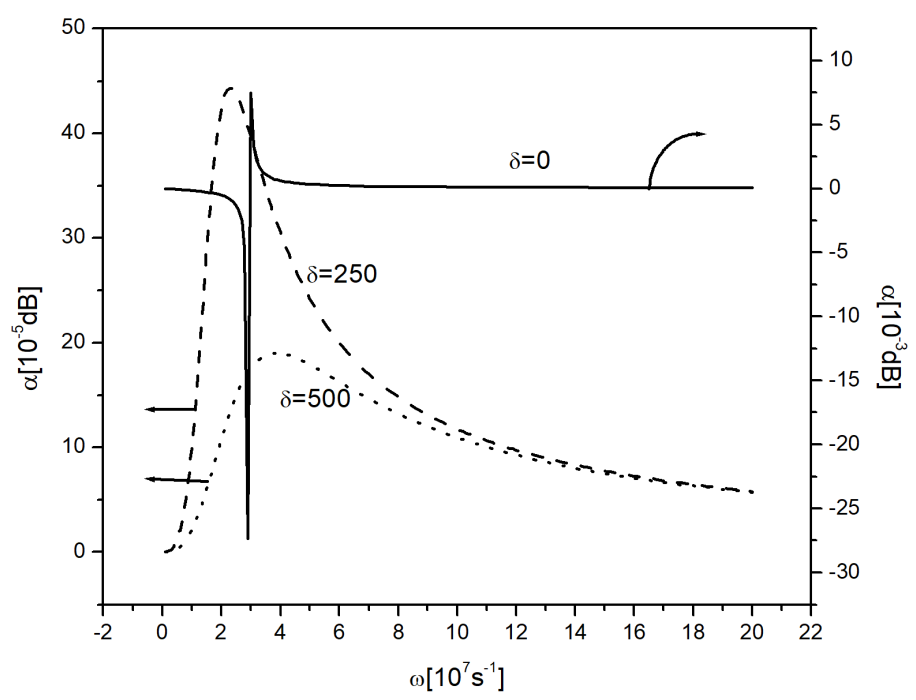

Fig. 3. $\alpha$ vs $\omega$ for different $\delta$ at $l=0.001$.

Figure $4 \mathrm{a}$ and $\mathrm{b}$ depicts the plot of acoustic gain $\alpha$ versus wave frequency $\omega$ in presence of the nanoparticle cluster at different values of non-dimensional parameter $l$ at $\delta=100$. The propagating acoustic mode is found amplifying in the frequency range $4.2 \times 10^{6}$ $7.7 \times 10^{6} \mathrm{~s}^{-1}$ (Fig. 4a) and attenuating at $2 \times 10^{7}-$ $2.4 \times 10^{7} \mathrm{~s}^{-1}$ (Fig. 4b). The maximum amplification is obtained at $\omega \approx 5.9 \times 10^{6} \mathrm{~s}^{-1}$ whereas maximum attenuation is obtained at $\omega \approx 2.3 \times 10^{7} \mathrm{~s}^{-1}$. Interestingly, the propagating shear acoustic mode is found stable in nature between these two ranges of frequencies $\left(7.7 \times 10^{6}-2 \times 10^{7} \mathrm{~s}^{-1}\right)$ discussed. We found that beyond $\omega \approx 2.5 \times 10^{7} \mathrm{~s}^{-1}$ this mode again starts amplifying with feeble gain constant and becomes stable afterwards. The non-dimensional parameter $l$, a function of particle density in the nanoparticle cluster is found responsible in reducing the gain and increasing the attenuation of the mode.

Figure 5 displays the variation of acoustic gain $\alpha$ with velocity ratio $\vartheta_{0} / \vartheta_{s}$ in presence of nanoparticle cluster using density gradient $\delta$ as parameter. For all values of $\delta$, the acoustic gain profiles have identical variations qualitatively with velocity ratio $\vartheta_{0} / \vartheta_{s}$. In these profiles, initially acoustic mode is attenuating, slowly attains maxima at a particular value of $\vartheta_{0} / \vartheta_{s}$ and then reduces to zero sharply. From this point onwards, this propagating acoustic mode becomes amplifying in nature whose amplification coefficient reaches to maximum more or less at the same value of $\vartheta_{0} / \vartheta_{s}$ at which we achieve maximum attenuation. If we increase the velocity ratio further this mode becomes stable. With the increase in inhomogeneity in the medium, one may shift the attenuation to amplification crossover point to lower values of velocity ratio. This figure infers that the dependences of acoustic gain $\alpha$ on varying velocity ratio $\vartheta_{0} / \vartheta_{s}$ is independent of non-dimensional parameter $l$ related to the existence of nanoparticle cluster within the semiconductor medium.
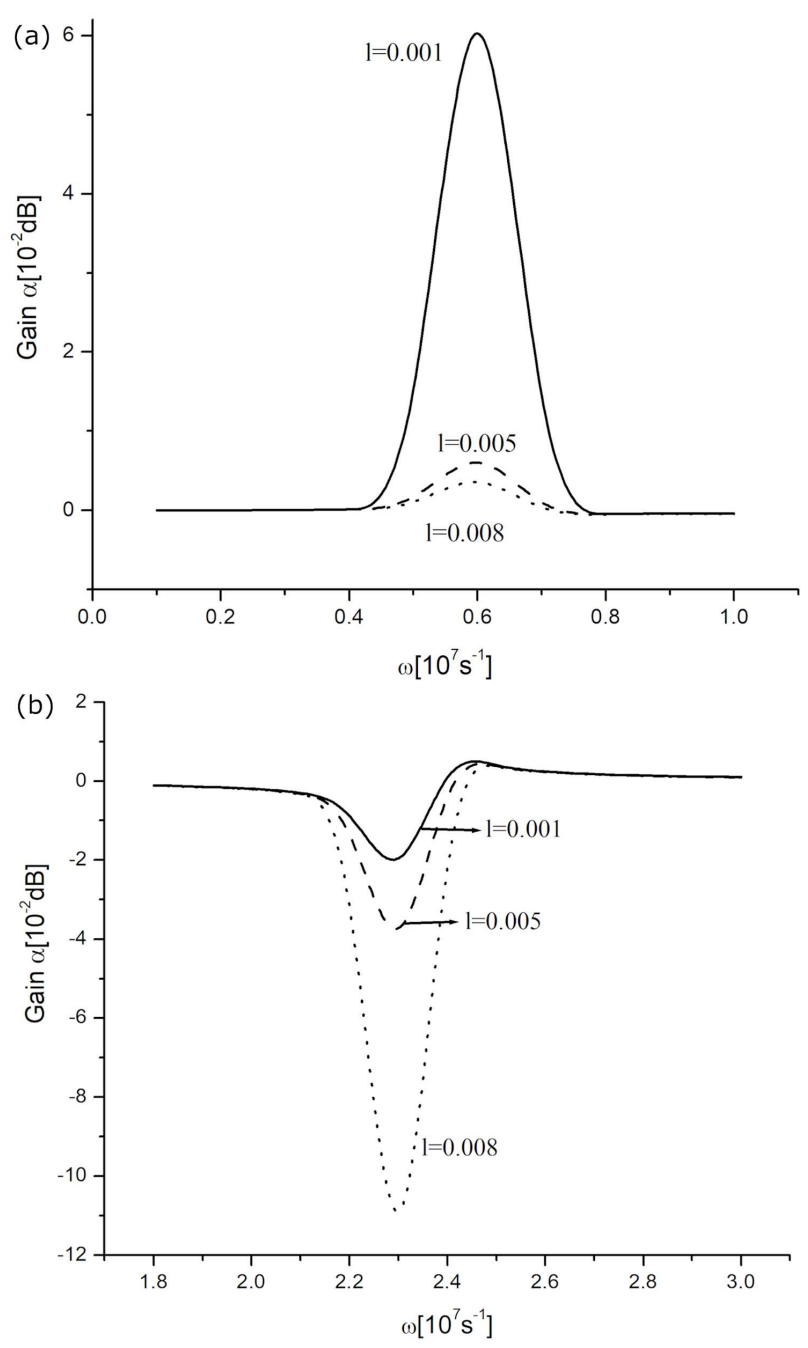

Fig. 4. $\alpha$ vs $\omega$ for different $l$ at $\delta=100$.

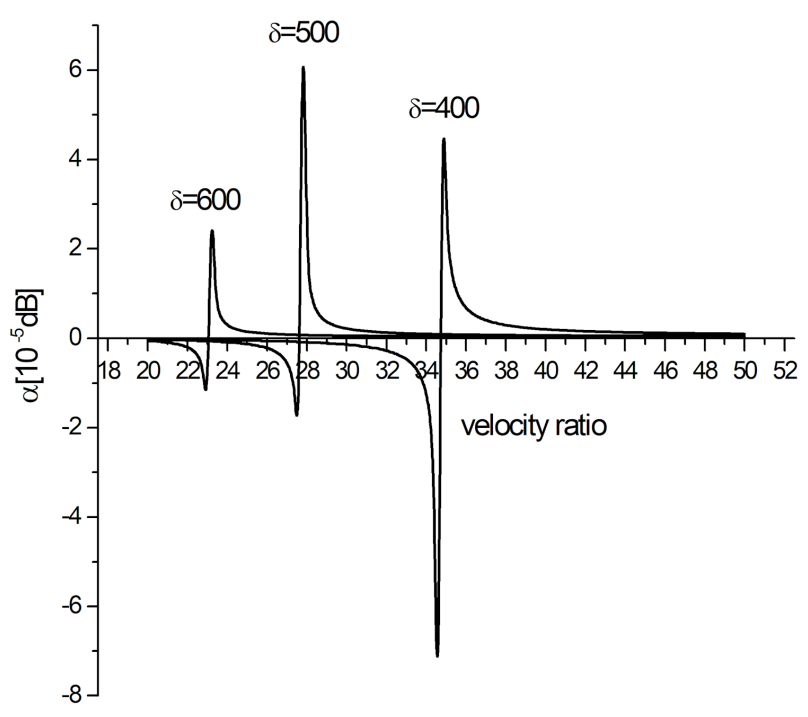

Fig. 5. $\quad \alpha$ vs $\vartheta_{0} / \vartheta_{s}$ for different $\delta$ at $l=0.001$. 
Precisely, the present study establishes that the presence of nanoparticle cluster and/or density gradient in cubic piezoelectric semiconductor medium are responsible for significant favourable modifications in the resonant interaction between acoustic phonons and plasmon modes. It is hoped that these outcomes would be useful in understanding the basic phonon-plasmon interaction phenomena in inhomogeneous semiconductor medium and tailoring the acoustic devices as per requirement.

\section{Acknowledgments}

One of the authors (P.D.) is thankful to her co-worker Apurva Muley for many valuable discussions.

\section{References}

[1] W. Chen, J.Z. Zhang, A.G. Joly, J. Nanosci. Nanotechnol. 4, 919 (2004).

[2] R.B. Vasiliev, D.N. Dirin, A.M. Gaskov, Russ. Chem. Rev. 80, 1139 (2011).

[3] M. Shakir, Siddhartha, G. Bhagavannarayana, M.A. Wahab, Chalcogen. Lett. 8, 435 (2011).

[4] W. Kim, J. Zide, A. Gossard, D. Klenov, S. Stemmer, A. Shakouri, A. Majumdar, Phys. Rev. Lett. 96, 045901 (2006).

[5] J.M. Zide, D.O. Klenov, S. Stemmer, A.C. Gossard, Appl. Phys. Lett. 87, 112102 (2005).

[6] J.K. Kawasaki, R. Timm, K.T. Delaney, E. Lundgren, A. Mikkelsen, C.J. Palmstrom, Phys. Rev. Lett. 107, 036806 (2011).

[7] B.D. Schultz, C.J. Palmstrom, Phys. Rev. B 73 , 241407(R) (2006).

[8] F. Lung, D.C. Marinescu, J. Phys. Condens. Matter 23, 365802 (2011).

[9] K.K. Choudhary, J. Nanopart. Res. 15, 1362 (2013).
[10] S. Ghosh, P. Dubey, Ind. J. Pure Appl. Phys. 55, 394 (2017).

[11] S. Ghosh, P. Dubey, Canad. J. Phys. 95, 95 (2017).

[12] A.R. Hutson, J.H. McFee, D.L. White, Phys. Rev. Lett. 7, 237 (1961).

[13] D.L. White, J. Appl. Phys. 33, 2547 (1962).

[14] A.R. Hutson, D.L. White, J. Appl. Phys. 33, 40 (1962).

[15] V.I. Pustovoit, Sov. Phys. Usp. 12, 105 (1969).

[16] L.C.M. Miranda, D. ter Haar, Rev. Bras. Fis. 2, 77 (1972).

[17] S.Y. Mensah, N.G. Mensah, V.W. Elloh, G.K. Banini, F. Sam, F.K.A. Allotey, Physica E 28, 500 (2005).

[18] K. Suresha, S.S. Kubakaddi, B.G. Mulimani, S.L. Lee, Physica E 33, 50 (2006).

[19] S. Ghosh, G.R. Sharma, P. Khare, M. Salimullah, Physica B 315, 163 (2004).

[20] A. Sharma, N. Nimje, N. Yadav, S. Ghosh, Int. J. Appl. Phys. 4, 37 (2014).

[21] S. Ghosh, A. Muley, Physica B 503, 75 (2016).

[22] S. Guha, S. Ghosh, N. Apte, Phys. Lett. A 71, 382 (1979).

[23] S. Ghosh, P. Khare, Acta Phys. Pol. A 109, 187 (2006).

[24] A. Sharma, N. Nimje, N. Yadav, S. Ghosh, Int. J. Adv. Res. Phys. Sci. 1, 19 (2014).

[25] S. Ghosh, A. Muley, Phys. Plasmas 23, 122115 (2016).

[26] W.P. Mason, Piezoelectric Crystals and Their Application to Ultrasonics, Van Nostrand, Princeton (NJ) 1950.

[27] M.C. Steele, B. Vural, Wave Interactions in Solid State Plasmas, Mc-Graw Hill, New York 1969.

[28] S. Jain, J. Parashar, Int. J. Nanosci. Nanotechnol. 2, 147 (2011). 